\title{
Observation of "Topological” Microflares in the Solar Atmosphere
}

\author{
Yurii V. Dumin ${ }^{1,2, *}$ and Boris V. Somov ${ }^{1}$ \\ ${ }^{1}$ Sternberg Astronomical Institute of Lomonosov Moscow State University, \\ 13 Universitetsky prosp., Moscow 119234, Russia \\ ${ }^{2}$ Space Research Institute of Russian Academy of Sciences
}

Keywords: magnetic reconnection — Sun: flares

Corresponding author: Yurii V. Dumin

dumin@pks.mpg.de,dumin@yahoo.com

* Currently in the Max Planck Institute for the Physics of Complex Systems, Dresden, Germany 


\section{OBSERVATIONAL DATA}

We report here on observation of the unusual kind of solar microflares, presumably associated with the so-called "topological trigger" of magnetic reconnection, which was theoretically suggested long time ago by Gorbachev et al. (1988) but has not been clearly identified so far by observations. The corresponding example is shown in the left-hand side of Figure 1: these frames were recorded by the Solar Optical Telescope (SOT) onboard Hinode satellite (Kosugi et al. 2007; Tsuneta et al. 2008) in the chromospheric line Ca II. ${ }^{1}$ At first sight, the bright loop flaring at 09:00:33 UT is just a magnetic field line connecting two sunspots. However, a closer inspection of the SDO HMI magnetogram (Pesnell et al. 2012) for the respective instant ${ }^{2}$ shows that the arc is anchored in the regions of the same polarity near the sunspot boundaries. So, it cannot be an ordinary magnetic-field line connecting the opposite magnetic poles.

08:58:33 UT

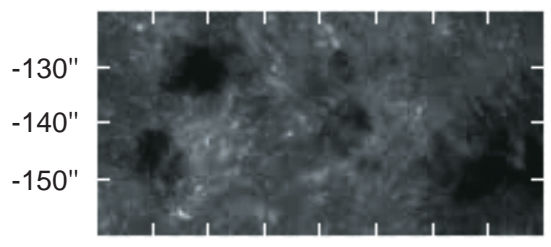

09:00:33 UT

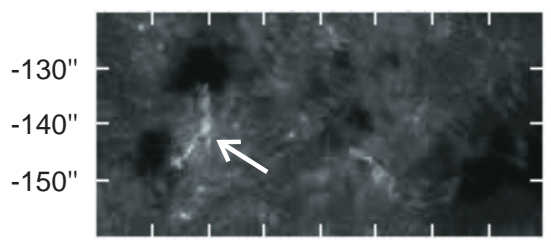

09:02:33 UT

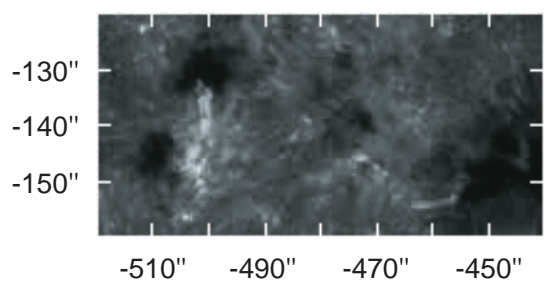

08:59:32 UT

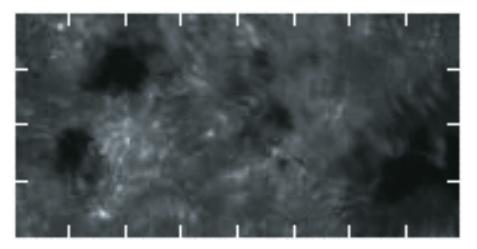

09:01:32 UT

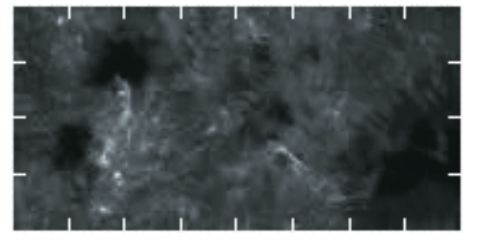

09:04:33 UT

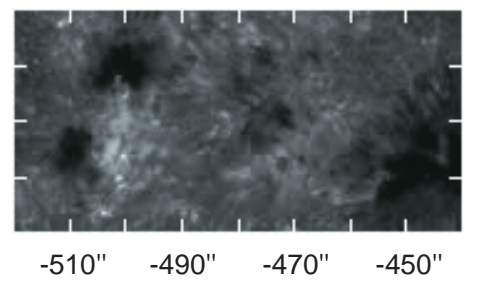

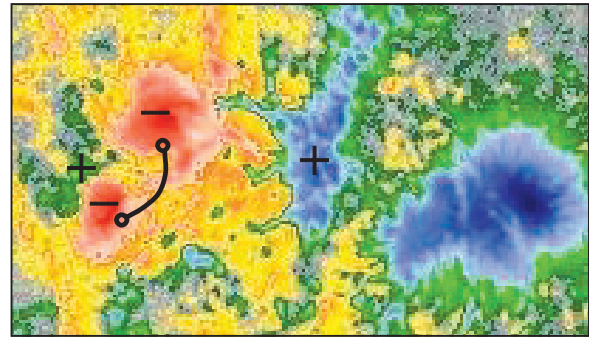

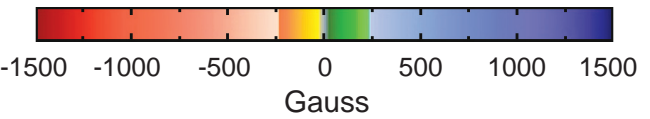

Figure 1. Left: Frames of emission in the Ca II line taken by Hinode SOT on 1 October 2014 at a few successive instants of time. Right: Magnetogram of the same region recorded by SDO HMI at 09:00:00 TAI. (Courtesy of NASA/SDO and the AIA, EVE, and HMI science teams.)

Yet another peculiar feature is that this arc flashes almost instantly as a thin strip and then begins to expand and decay, while typical the chromospheric flares in Ca II line are much wider and propagate progressively in space.

\section{THEORETICAL INTERPRETATION}

A qualitative explanation of the observed phenomenon can be given by the above-mentioned model of topological trigger. Namely, there are such configurations of the magnetic sources (e.g. point-like magnetic poles) on the surface of photosphere that their tiny displacements result in the formation and fast motion of a 3D null point along the arc located well above the plane of the sources. So, such a null point can quickly ignite a magnetic reconnection along the entire its trajectory. Pictorially, this can be presented as flipping the so-called two-dome magnetic-field structure (which is just the reason why such mechanism of reconnection was called topological). The general mathematical criteria for its onset can be found in paper by Gorbachev et al. (1988), while our recent numerical simulations will be published elsewhere. In brief, the most important prerequisite for the development of topological instability in the two-dome structure is a cruciform arrangement of the magnetic sources in its base. As can be seen in the right panel of Figure 1, this condition is really satisfied in the case under consideration.

\footnotetext{
1 The data were taken from the Hinode QL Movie Archive at http://hinode.nao.ac.jp/en/for-researchers/qlmovies/

2 The data were taken from the Joint Science Operations Center (JSOC) at http://jsoc.stanford.edu/HMI/hmiimage.html
} 
Let us mention that previous attempts to identify the topological trigger in the solar atmosphere were concentrated mostly on the large flares (e.g. Oreshina, A. et al. 2012; Oreshina, I. \& Somov 2009). As a result, it was found that the magnetic-field configuration required for the development of topological trigger is sometimes formed just before the onset of the powerful flares. Unfortunately, it was impossible to identify the trajectory of the 3D null point, because the entire picture was strongly obscured by the intense electric currents generated immediately in the spot of reconnection. The advantage of the present study is that (1) we analyzed the microflares, which are governed mostly by the external (photospheric) magnetic sources, and (2) we employed observations in the chromospheric Ca II line, which is emitted by a moderately heated plasma and, thereby, can trace the initial stage of reconnection.

We are grateful to A. V. Getling, I. V. Oreshina, and A. V. Oreshina for valuable discussions and advices. Hinode is a Japanese mission developed and launched by ISAS/JAXA, with NAOJ as domestic partner and NASA and STFC (UK) as international partners. It is operated by these agencies in co-operation with ESA and NSC (Norway).

\section{REFERENCES}

Gorbachev, V. S., Kelner, S. R., Somov, B. V., \& Shvarts, A. S. 1988, Soviet Ast., 32, 308

Kosugi, T., Matsuzaki, K., Sakao, T., et al. 2007, SoPh, 243,3

Oreshina, A. V., Oreshina, I. V., \& Somov, B. V. 2012, A\&A, 538, A138
Oreshina, I. V. \& Somov, B. V. 2009, Ast. Lett., 35, 207

Pesnell, W. D., Thompson, B. J., \& Chamberlin, P. C. 2012, SoPh, 275, 3

Tsuneta, S., Suematsu, Y., Ichimoto, K., et al. 2008, SoPh, 249, 167 